\title{
P-0925: Performance of stepwise screening methods in identifying those at high risk of type 2 diabetes in an Iranian population
}

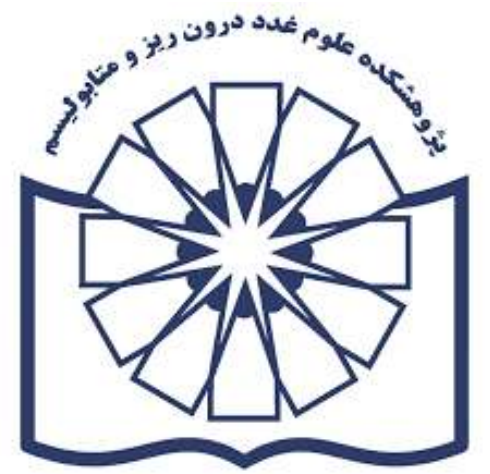

\author{
Authors: Mojtaba Lotfaliany (1), Davood Khalili (2), Farzad Hadaegh (3), Fereidoun Azizi (3) \\ 1.The University of Melbourne, Australia \\ 2.Department of Biostatistics and Epidemiology, Research Institute for Endocrine Sciences, Shahid Beheshti University of Medical \\ Sciences, Tehran, Iran \\ 3.Research Institute for Endocrine Sciences, Shahid Beheshti University of Medical Sciences, Tehran, Iran
}

\section{Background}

Recent evidence recommended stepwise screening methods for identifying individuals at high risk of type 2 diabetes to be recruited in the lifestyle intervention programs for prevention of the disease.

\begin{abstract}
Aims
This study aims to assess the performance of different stepwise screening methods that combine non-invasive measurements with labbased measurements for identifying those with 5 -years incident type 2 diabetes.
\end{abstract}

\section{Method}

3037 participants aged $\geq 30$ years without diabetes at baseline in the Tehran Lipid and Glucose study were followed. Thirty-two stepwise screening methods were developed by combining a non-invasive measurement (an anthropometric measurement (waist-to-height ratio) or a score based on a non-invasive risk score (Australian Type 2 Diabetes Risk Assessment Tool)) with a lab-based measurement (different cut-offs of fasting plasma glucose or predicted risk based on three lab-based prediction models (Saint Antonio, Framingham Offspring Study, and the Atherosclerosis Risk in Communities)). The validation, calibration, and usefulness of labbased prediction models were assessed before developing the stepwise screening methods. Cut-offs were derived either based on previous studies or decision-curve analyses.

\section{Results}

203 participants developed diabetes in 5 years. Lab-based risk prediction models had good discrimination power (AUCs: 0.80-0.83), achieved acceptable calibration and net benefits after recalibration for population's characteristics and were useful in a wide range of risk thresholds $(5 \%-21 \%)$.
Different stepwise methods had sensitivity ranged 20\%-68\%, specificity 70\%-98\%, and PPV 14\%-46\%; they identified 3\%-33\% of the screened population eligible for preventive interventions.

\section{Discussion}

Lab-based prediction models are valid tools for prediction of the risk of type 2 diabetes after calibration; and using them in combination with non-invasive measurements can form effective stepwise screening methods with acceptable sensitivity and specificity in Iranian population. Using stepwise methods can also eliminate the need for lab measurements in about half of the screened population and limit the proportion of the population who need preventive interventions.

Table. Performance of stepwise methods in identifying individuals with 5-year incident type 2 diabetes.

\begin{tabular}{|c|c|c|c|c|}
\hline Step 1 & Step 2 & $\begin{array}{l}\text { Sensitivity\% } \\
(95 \% \mathrm{Cl})\end{array}$ & $\begin{array}{l}\text { Specificity } \\
\%(95 \% \mathrm{Cl})\end{array}$ & $\begin{array}{l}\text { PPV \% } \\
(95 \% \mathrm{Cl})\end{array}$ \\
\hline $\mathrm{WHtR} \geq 0.55$ & $\overline{F P G} \geq 5.0 \mathrm{mmol} / \mathrm{L}$ & $66(59 ; 72)$ & $73(71 ; 75)$ & $15(13 ; 17)$ \\
\hline WHtR $\geq 0.55$ & $\mathrm{FPG} \geq 5.3 \mathrm{mmol} / \mathrm{L}$ & $58(51 ; 65)$ & $83(82 ; 85)$ & $20(17 ; 23)$ \\
\hline WHtR $\geq 0.55$ & $\mathrm{FPG} \geq 5.5 \mathrm{mmol} / \mathrm{L}$ & $47(40 ; 54)$ & $91(90 ; 92)$ & $27(23 ; 32)$ \\
\hline WHtR $\geq 0.55$ & $\mathrm{FPG} \geq 6.1 \mathrm{mmol} / \mathrm{L}$ & $20(14 ; 26)$ & $98(98 ; 99)$ & $46(35 ; 56)$ \\
\hline WHtR $\geq 0.55$ & $S A \geq 7.5 \%$ & $60(52.5 ; 66)$ & $84(82 ; 85)$ & $21(17 ; 24)$ \\
\hline $\mathrm{WHtR} \geq 0.55$ & $S A \geq 10 \%$ & $53(46 ; 60)$ & $88(87 ; 89)$ & $24(20 ; 28)$ \\
\hline $\mathrm{WHtR} \geq 0.55$ & $S A \geq 15 \%$ & $41(34 ; 48)$ & $92(91 ; 93)$ & $27(22 ; 32)$ \\
\hline WHtR $\geq 0.55$ & $S A \geq 20 \%$ & $33(27 ; 40)$ & $95(94 ; 96)$ & $33(26 ; 39)$ \\
\hline $\mathrm{WHtR} \geq 0.55$ & $A R I C \geq 7.5 \%$ & $59(52 ; 66)$ & $83(82 ; 85)$ & $20(17 ; 24)$ \\
\hline WHtR $\geq 0.55$ & $A R I C \geq 10 \%$ & $53(45 ; 60)$ & $88(87 ; 89)$ & $24(20 ; 28)$ \\
\hline $\mathrm{WHtR} \geq 0.55$ & $A R I C \geq 15 \%$ & $42(35 ; 49)$ & $93(92 ; 94)$ & $29(24 ; 35)$ \\
\hline $\mathrm{WHtR} \geq 0.55$ & $A R I C \geq 20 \%$ & $35(28 ; 42)$ & $95(95 ; 96)$ & $35(28 ; 41)$ \\
\hline WHtR $\geq 0.55$ & FOS $\geq 7.5 \%$ & $55(48 ; 62)$ & $86(85 ; 87)$ & $22(18 ; 25)$ \\
\hline $\mathrm{WHtR} \geq 0.55$ & $\mathrm{FOS} \geq 10 \%$ & $51(44 ; 58.5)$ & $89(88 ; 90)$ & $25(21 ; 30)$ \\
\hline WHtR $\geq 0.55$ & FOS $\geq 15 \%$ & $41(34 ; 48)$ & $92(91 ; 93)$ & $27(22 ; 32)$ \\
\hline $\mathrm{WHtR} \geq 0.55$ & FOS $\geq 20 \%$ & $36(29 ; 43)$ & $94(93 ; 95)$ & $31(25 ; 37)$ \\
\hline AUSDRISC > 15 & $\mathrm{FPG} \geq 5.0 \mathrm{mmol} / \mathrm{L}$ & $68(62 ; 75)$ & $70(68 ; 71)$ & $14(12 ; 16)$ \\
\hline AUSDRISC > 15 & $\mathrm{FPG} \geq 5.3 \mathrm{mmol} / \mathrm{L}$ & $61(54 ; 68)$ & $81(79 ; 82)$ & $18(16 ; 22)$ \\
\hline AUSDRISC > 15 & $\mathrm{FPG} \geq 5.5 \mathrm{mmol} / \mathrm{L}$ & $48(41 ; 55)$ & $89(88 ; 91)$ & $25(20 ; 29)$ \\
\hline AUSDRISC > 15 & $\mathrm{FPG} \geq 6.1 \mathrm{mmol} / \mathrm{L}$ & $22(16 ; 28)$ & $98(97 ; 98)$ & $43(34 ; 53)$ \\
\hline AUSDRISC > 15 & $S A \geq 7.5 \%$ & $60(53 ; 67)$ & $82(81 ; 83)$ & $19(16 ; 22)$ \\
\hline AUSDRISC > 15 & $S A \geq 10 \%$ & $53(46 ; 60)$ & $87(85 ; 88)$ & $22(18 ; 26)$ \\
\hline AUSDRISC > 15 & $S A \geq 15 \%$ & $41(35 ; 48)$ & $91(90 ; 92)$ & $26(21 ; 31)$ \\
\hline AUSDRISC > 15 & $S A \geq 20 \%$ & $34(28 ; 41)$ & $95(94 ; 95)$ & $32(25 ; 38)$ \\
\hline AUSDRISC > 15 & $A R I C \geq 7.5 \%$ & $61(54 ; 67.5)$ & $82(81 ; 84)$ & $20(16 ; 23)$ \\
\hline AUSDRISC > 15 & ARIC $\geq 10 \%$ & $52(45 ; 59)$ & $87(86 ; 88)$ & $22(18 ; 26)$ \\
\hline AUSDRISC > 15 & $A R I C \geq 15 \%$ & $42(35 ; 49)$ & $92(91 ; 93)$ & $28(23 ; 33)$ \\
\hline AUSDRISC > 15 & $A R I C \geq 20 \%$ & $35(29 ; 42)$ & $95(94 ; 96)$ & $34(27 ; 40)$ \\
\hline AUSDRISC > 15 & $\mathrm{FOS} \geq 7.5 \%$ & $57(50 ; 63)$ & $84(83 ; 86)$ & $21(17 ; 24)$ \\
\hline AUSDRISC > 15 & $\mathrm{FOS} \geq 10 \%$ & $53(46 ; 60)$ & $88(87 ; 89)$ & $24(20 ; 29)$ \\
\hline AUSDRISC > 15 & FOS $\geq 15 \%$ & $42(34.5 ; 49)$ & $92(91 ; 93)$ & $26(21 ; 31)$ \\
\hline AUSDRISC > 15 & FOS $\geq 20 \%$ & $36(29 ; 43)$ & $94(93 ; 95)$ & $29(23.5 ; 35)$ \\
\hline
\end{tabular}

